\title{
Genetic alterations of triple negative breast cancer by targeted next-generation sequencing and correlation with tumor morphology
}

\author{
Paul S Weisman ${ }^{1,3}$, Charlotte KY Ng${ }^{1,3}$, Edi Brogi ${ }^{1}$, Rachel E Eisenberg ${ }^{1}$, Helen H Won ${ }^{1}$, \\ Salvatore Piscuoglio ${ }^{1}$, Maria R De Filippo ${ }^{1}$, Rafael Ioris ${ }^{1}$, Muzaffar Akram ${ }^{1}$, Larry Norton ${ }^{2}$, \\ Britta Weigelt ${ }^{1}$, Michael F Berger ${ }^{1}$, Jorge S Reis-Filho ${ }^{1}$ and Hannah Y Wen ${ }^{1}$
${ }^{1}$ Department of Pathology, Memorial Sloan Kettering Cancer Center, New York, NY, USA and ${ }^{2}$ Department of Medicine, Memorial Sloan Kettering Cancer Center, New York, NY, USA

\begin{abstract}
Triple negative breast cancer represents a heterogeneous group of breast carcinomas, both at the histologic and genetic level. Although recent molecular studies have comprehensively characterized the genetic landscape of these tumors, few have integrated a detailed histologic examination into the analysis. In this study, we defined the genetic alterations in 39 triple negative breast cancers using a high-depth targeted massively parallel sequencing assay and correlated the findings with a detailed morphologic analysis. We obtained representative frozen tissue of primary triple negative breast cancers from patients treated at our institution between 2002 and 2010. We characterized tumors according to their histologic subtype and morphologic features. DNA was extracted from paired frozen primary tumor and normal tissue samples and was subjected to a targeted massively parallel sequencing platform comprising 229 cancer-associated genes common across all experiments. The average number of non-synonymous mutations was 3 (range $0-10)$ per case. The most frequent somatic alterations were mutations in TP53 (74\%) and PIK3CA (10\%) and MYC amplifications (26\%). Triple negative breast cancers with apocrine differentiation less frequently harbored TP53 mutations (25\%) and MYC gains ( $0 \%$ ), and displayed a high mutation frequency in PIK3CA and other PI3K signaling pathway-related genes $(75 \%)$. Using a targeted massively parallel sequencing platform, we identified the key somatic genetic alterations previously reported in triple negative breast cancers. Furthermore, our findings show that triple negative breast cancers with apocrine differentiation constitute a distinct subset, characterized by a high frequency of PI3K pathway alterations similar to luminal subtypes of breast cancer.

Modern Pathology (2016) 29, 476-488; doi:10.1038/modpathol.2016.39; published online 4 March 2016
\end{abstract}

Triple negative breast cancer represents a heterogenous group of breast carcinomas that lack expression of estrogen receptor, progesterone receptor, and HER2. As a group, triple negative breast cancers have an aggressive clinical course and a poor prognosis. ${ }^{1}$ Morphologically, the majority of the triple negative breast cancers are high-grade invasive ductal carcinomas of no special type, and are associated with tumor necrosis, pushing borders, and prominent tumor-infiltrating lymphocytes. ${ }^{2,3}$

Correspondence: Dr HY Wen, MD, PhD, Department of Pathology, Memorial Sloan Kettering Cancer Center, 1275 York Avenue, New York, NY 10065, USA.

E-mail: weny@mskcc.org

Part of this work was presented at United States and Canadian Academy of Pathology (USCAP) 103rd Annual Meeting in San Diego March 2014.

${ }^{3}$ These authors contributed equally to this work.

Received 24 November 2015; revised 13 January 2016; accepted 15 January 2016; published online 4 March 2016
Some special histologic subtypes also show a triple negative phenotype, including most metaplastic carcinomas, a subset of carcinomas with apocrine differentiation, adenoid cystic carcinomas, secretory carcinomas, and acinic cell carcinomas. ${ }^{4-12}$ Given the lack of expression of estrogen receptor, progesterone receptor, and HER2, chemotherapy is currently the only option for systemic therapy in patients with triple negative breast cancer.

Recent studies have described the comprehensive molecular genetic landscape of human breast cancers using whole genome/exome sequencing, RNA sequencing, and Affymetrix SNP array analyses. ${ }^{13-15}$ Recurrent somatic mutations with greater than $10 \%$ frequency across all breast cancer subtypes were found in only three genes: TP53, PIK3CA, and GATA3, which occurred at $37 \%, 36 \%$, and $11 \%$, respectively. ${ }^{13}$ The patterns of somatic mutations among the intrinsic breast cancer subtypes were different. Basal-like and triple negative breast 
cancers showed a high frequency of TP53 mutation $(80 \%)$, whereas only $12 \%$ of luminal A and $29 \%$ luminal B tumors harbored TP53 mutations. PIK3CA is the most frequently mutated gene in luminal A (45\%) and luminal B (29\%) breast cancer. Although PIK3CA was the second most frequently mutated gene in triple negative breast cancers, the frequency of PIK3CA mutation in triple negative breast cancers was lower than that in luminal breast cancer, at $9-10 \% .{ }^{13,14}$ While such studies have increased our understanding of the genetic landscape of triple negative breast cancer relative to other breast cancer subtypes, few have integrated a molecular genetic analysis of triple negative breast cancers with a detailed histologic evaluation.

In this study, we characterized the genetic alterations in a group of triple negative breast cancers and correlated the results with a detailed morphologic analysis. We utilized the Integrated Mutation Profiling of Actionable Cancer Targets (MSK-IMPACT) platform, ${ }^{16,17}$ a targeted next-generation sequencing assay targeting all coding regions and selected regulatory and intronic regions of 229 of the most common cancer genes.

\section{Materials and methods}

\section{Patient Selection}

This study was approved by the Memorial Sloan Kettering Cancer Center Institutional Review Board. Informed patient consent was obtained as appropriate, following the protocols approved by the Memorial Sloan Kettering Cancer Center Institutional Review Board. We identified patients with primary triple negative breast cancer treated at our institution between 2002 and 2010 through a search of the institution database. Triple negative breast cancer was defined as invasive breast carcinoma with estrogen receptor and progesterone receptor staining in less than $1 \%$ of the tumor cells by immunohistochemistry and no HER2 overexpression (defined as negative (0 to $1+$ ) or equivocal $(2+)$ staining by immunohistochemistry and no amplification by fluorescence in situ hybridization), in accordance with the American Society of Clinical Oncology/College of American Pathologists guidelines. ${ }^{18-21}$ We retrieved available frozen samples of paired primary tumor and normal tissue for the study. Patients who received neoadjuvant chemotherapy were excluded from the study.

\section{Clinicopathological Review}

Clinicopathological data for each patient, including age at diagnosis, BRCA1 germline mutation status, tumor characteristics (size, grade, special histologic subtypes, and morphologic features-details below), lymph node involvement, distant metastases, length of follow-up, and survival status, were recorded. All available slides were reviewed by two pathologists (HYW and REE) to assess the histologic features.
Special histologic subtypes (metaplastic carcinoma and carcinoma with apocrine differentiation) were defined according the 2012 WHO classification. ${ }^{22,23}$ Apocrine differentiation was defined as nuclear enlargement with prominent nucleoli and abundant, granular, eosinophilic cytoplasm. ${ }^{23}$ Metaplastic carcinoma was defined as carcinoma with squamous differentiation, spindle cell morphology, or mesenchymal elements. ${ }^{22}$ Other morphologic features commonly seen in triple negative breast cancer, such as a large central acellular zone of necrosis or fibrosis and tumorinfiltrating lymphocytes were also recorded. Large central acellular zone morphology was defined as the presence of a large, centrally located paucicellular or acellular area occupying $>30 \%$ of the tumor area and not associated with extensive coagulative necrosis, squamous debris, or overt cartilaginous matrix production. ${ }^{24}$ Tumor-infiltrating lymphocytes were scored as a percentage of the stromal areas occupied by mononuclear cells including lymphocytes and plasma cells, excluding granulocytes and other polymorphonuclear leukocytes. ${ }^{25}$ Tumorinfiltrating lymphocytes were scored according to the recommendations by the International Tumor infiltrating Lymphocytes Working Group. ${ }^{25}$ Tumors with $\geq 50 \%$ tumor-infiltrating lymphocytes were classified as having prominent tumor-infiltrating lymphocytes. ${ }^{26}$ Triple negative breast cancers with none of these special features were classified as triple negative breast cancer not otherwise specified.

\section{Immunohistochemistry for Androgen Receptor}

Immunohistochemical staining was performed on representative 4-micron-thick sections in cases with available paraffin blocks using antibodies against the androgen receptor (DAKO AR441 clone; 1:75 dilution; pretreatment with citric buffer, $\mathrm{pH}$ 6.2; HRP detection; DAB chromogen). Scoring of androgen receptor paralleled the American Society of Clinical Oncology/College of American Pathologists guidelines for estrogen receptor and progesterone receptor. Nuclear staining for androgen receptor in $\geq 1 \%$ of tumor cells was considered positive.

\section{DNA Extraction}

DNA was extracted from paired frozen primary tumor ( $>75 \%$ tumor content) and normal tissue samples using the DNeasy Blood and Tissue Kit (QIAGEN). DNA samples were subjected to targeted massively parallel sequencing using the MSK-IMPACT sequencing assay (details below). ${ }^{16}$

\section{The MSK-IMPACT Assay}

Deep targeted sequencing of key cancer-associated genes was performed using the MSK-IMPACT 
assay. ${ }^{16}$ In this assay, target-specific oligonucleotide probes were designed to capture all protein-coding exons of the most common cancer-related genes (229 genes at the time of this study; Supplementary Table 1) for hybrid selection (Agilent SureSelect or Nimblegen SeqCap) as previously described. ${ }^{17,27}$ For 26 samples (13 tumor/normal pairs), barcoded sequence libraries (Illumina TruSeq) were prepared using $500 \mathrm{ng}$ of input tumor or matched normal DNA according to the manufacturer's instructions. Libraries were pooled at equimolar concentrations (100 ng per tumor library and $50 \mathrm{ng}$ per normal library) for a single exon capture reaction (Agilent SureSelect) as previously described. For the remaining samples, barcoded sequence libraries were prepared using $250 \mathrm{ng}$ of input DNA using a hybrid protocol on the basis of the NEBNext DNA Library Prep Kit (New England Biolabs). Manufacturer's instructions were followed with two substitutions: we used NEXTflex barcoded adapters (Bio Scientific) and HiFi DNA polymerase (Kapa Biosystems). Libraries were pooled at $100 \mathrm{ng}$ per tumor library and $50 \mathrm{ng}$ per normal library and captured using custom biotinylated DNA probes (Nimblegen SeqCap). To prevent off-target hybridization in all capture reactions, we spiked in a pool of blocker oligonucleotides complementary to the full sequences of all barcoded adaptors (to a final total concentration of $10 \mu \mathrm{M})$. Hybridized DNA was sequenced on an Illumina HiSeq 2000 to generate paired-end 75-bp reads.

Data were demultiplexed using CASAVA, and reads were aligned to the reference human genome (hg19) using the Burrows-Wheeler Alignment tool. ${ }^{28}$ Local realignment and quality score recalibration were performed using the Genome Analysis Toolkit (GATK) according to GATK best practices. ${ }^{29} \mathrm{We}$ achieved mean exon sequence coverage of $507 \times$ $(678 \times$ for all tumor samples and $348 \times$ for all normal samples). Deep sequencing ensured sensitivity for detecting mutations in multiclonal and stromaadmixed samples and enabled accurate determination of mutation allele frequencies.

Sequence data were analyzed to identify three classes of somatic alterations: single-nucleotide variants, small insertions/deletions (indels), and copy number alterations, adopting the CLIA-compliant analysis methods as previously published. ${ }^{16}$ Singlenucleotide variants were called using MuTect, ${ }^{30}$ and retained if the variant allele frequency in the tumor was $>5$ times that in the matched normal. Indels were called using the SomaticIndelDetector tool in GATK. $^{29}$ Somatic single-nucleotide variants and small indels with variant allele frequency $<5 \%$ were excluded. All candidate mutations and indels were further reviewed manually using the Integrative Genomics Viewer. ${ }^{31}$ The mean sequence coverage was calculated using the DepthOfCoverage tool in GATK and was used to compute copy number. Copy number gains and losses were determined by calculating the tumor to normal ratio in normalized sequence coverage across all target exons following a loss normalization to adjust for G/C content. Ratios $<0.5$ were considered as deletions, between 0.5 and 0.7 were considered copy number losses, between 1.3 and 1.5 were considered copy number gains, and ratios $>1.5$ were considered as amplifications.

\section{Mutation Significance Analysis}

Mutations resulting in frameshift insertions or deletions (indels) and those involving splice sites or resulting in nonsense mutations were considered pathogenic. We assessed the functional effects of missense mutations using CHASM, ${ }^{32}$ FATHMM, ${ }^{33}$ Mutation Assessor, ${ }^{34}$ Mutation Taster, ${ }^{35}$ and Polyphen-2. ${ }^{36}$ Mutations predicted to be deleterious or cancer drivers by at least two algorithms were considered potential driver mutations. Deleterious or pathogenic classifications from Mutation Taster and Polyphen-2 were counted as one as these two algorithms frequently returned the same predictions and were underpinned by similar bioinformatic principles. In-frame indels were considered pathogenic if predicted to be deleterious by either PROVEAN $^{37}$ or Mutation Taster.

\section{Sanger Sequencing}

Putative somatic mutations in selected genes of interest identified by MSK-IMPACT sequencing were further investigated by Sanger sequencing. Primer sets that amplify mutated exons of the selected genes were designed as previously described $^{38}$ and are available in Supplementary Table 2. PCR amplification of $5 \mathrm{ng}$ of genomic DNA was performed using the AmpliTaq 360 Master Mix Kit (Life Technologies) on a Veriti Thermal Cycler (Life Technologies) as previously described. ${ }^{38}$ PCR fragments were purified (ExoSAP-IT, Affymetrix) and sequenced on an ABI 3730 capillary sequencer using the ABI BigDye Terminator chemistry (v3.1, Life Technologies). Sequences of the forward and reverse strands were analyzed using MacVector software (MacVector), ${ }^{38}$ and all analyses were performed in duplicates. Representative Sanger sequencing chromatograms of validated mutations are shown in Supplementary Figure 1. Of the 26 putative somatic mutations selected, 24 were successfully validated (Supplementary Table 3), resulting in an overall validation rate of $92 \%$, providing confidence to include the remaining putative mutations not subjected to Sanger sequencing. The two variants that failed Sanger sequencing were removed from further analyses.

\section{Statistical Analysis}

Statistical analysis of clinicopathologic data was performed using a two-tailed student $t$-test for 
continuous variables and a Fisher's exact test for categorical variables. Mutational frequencies and GISTIC2 copy number data for the triple negative breast cancers from the Cancer Genome Atlas were obtained from the publication data portal (https:// the Cancer Genome Atlas -data.nci.nih.gov/docs/ publications/brca_2012/). For the statistical analysis comparing proportion of cases affected by nonsynonymous mutational or copy number alterations (amplifications and deletions, or 2 and -2 in the GISTIC2 copy number data) in a particular gene between the current cohort and triple negative breast cancers from the Cancer Genome Atlas and between subtypes of triple negative breast cancers within the current cohort, Fisher's exact test for nonparametric variables was used, with all probabilities reported as two-tailed. $P$-values $<0.05$ were considered statistically significant. All analyses were performed using R v3.0.2.

\section{Results}

\section{Clinicopathologic Characteristics of the Patients}

Clinicopathological characteristics of the patients are summarized in Table 1. All patients were female. The median age at diagnosis was 43 years (range 28-78). Fourteen patients had genetic testing for $B R C A$ germline mutations and nine were found to be BRCA1 germline mutation carriers. The median tumor size was $2.9 \mathrm{~cm}$ (range 1.2-8.5). Most cases had high tumor grade (histologic grade III and nuclear grade III). Four (10\%) cases had apocrine differentiation, 1 (3\%) case was a metaplastic carcinoma with chondroid matrix production, and the remaining $34(87 \%)$ triple negative breast cancers were invasive ductal carcinoma of no special type as defined by the WHO classification criteria (Figure 1). ${ }^{39}$ Of these, six (15\%) had a large central acellular zone and six (15\%) had prominent tumorinfiltrating lymphocytes (Figure 1). Cases with a large central acellular zone and prominent tumorinfiltrating lymphocytes did not overlap. The remaining 22 triple negative breast cancers (56\%) had no special morphologic features (triple negative breast cancer not otherwise specified).

Lymph node involvement at presentation was seen in $19(49 \%)$ patients, and $17(44 \%)$ patients developed distant metastases. The most common site of distant metastasis was lung $(n=11)$, followed by bone $(n=6)$, brain $(n=5)$, and liver $(n=2)$. Six patients had distant metastases to multiple sites. Sixteen $(41 \%)$ patients died from their breast cancer at a median follow-up interval of 62 months (range 10-143).

\section{Immunohistochemistry}

Paraffin blocks were available in 30 of 39 cases, including 17 triple negative breast cancers not otherwise specified, 5 tumors with prominent tumor-infiltrating lymphocytes, 4 tumors with a large central acellular zone, and all 4 triple negative breast cancers with apocrine differentiation. Four tumors were positive for androgen receptor by immunohistochemistry; three of these were triple negative breast cancers with apocrine differentiation and the remaining case was triple negative breast cancer not otherwise specified (75 vs 4\%; $P=0.0038)$.

\section{Somatic Mutations and Copy Number Alterations}

The average depth of sequencing was $678 \times$ for tumor samples and $348 \times$ for normal samples. Among the 229 genes profiled across our entire cohort, the average number of non-synonymous mutations per case was 3.33 (range $0-10$ ), significantly higher than that of the triple negative breast cancers in the Cancer Genome Atlas data set (mean 2.37, range $0-7, P=0.008737$, Mann-Whitney U test). The most frequently mutated genes in our cohort were TP53 (29 cases, 74\%), followed by KMT2D (also known as MLL2, 6 cases, 15\%) and PIK3CA (4 cases, $10 \%$ ) (Figure 2). While the frequencies of TP53 and PIK3CA mutations are similar to those of triple negative breast cancers reported by the Cancer Genome Atlas and other studies, ${ }^{13,14}$ mutations in MLL2 were significantly more frequent in our cohort than in triple negative breast cancers from the Cancer Genome Atlas (MLL2: 6/39 (15\%) vs 2/78 cases (3\%), $P=0.0162$, Fisher's exact test). Other statistically significant differences between our cohort and the cohort of triple negative breast cancers from the Cancer Genome Atlas include the frequency of KDM6A, JAK1, and PIK3R1 mutations, each present in three cases $(8 \%)$ in our cohort in contrast to none of the cases in the Cancer Genome Atlas cohort $(P=0.0351$, Fisher's exact tests).

The overall pattern of copy number alterations in this cohort is similar to that in the triple negative breast cancers from the Cancer Genome Atlas breast cancer study (Figure 3). The most frequent copy number alterations in this cohort were amplifications of MYC (10 cases, $26 \%$ ), MCL1 (6 cases, 15\%), and DDR2 (6 cases, $15 \%$ ) and deletions of CDKN2A/ CDKN2B (2 cases, 5\%) (Figure 2). Amplifications of PIK3C2G were more frequent in our cohort than that of the triple negative breast cancers in the Cancer Genome Atlas cohort (4/39, $10 \%$ vs 0/78, 0\%, $P=0.011$, Fisher's exact test).

\section{Genetic Alterations by Histologic Subtypes and Morphologic Features}

We next performed an exploratory, hypothesisgenerating analysis of the repertoire of somatic genetic alterations found in triple negative breast cancers according to histologic subtypes and specific morphologic features (Table 2 and Figure 4). 
Table 1 Clinicopathological characteristics of 39 cases

\begin{tabular}{|c|c|c|c|c|c|c|}
\hline & $\begin{array}{l}\text { All cases } \\
(\mathrm{n}=39)\end{array}$ & $\begin{array}{l}\text { TNBC NOS } \\
(\mathrm{n}=22)\end{array}$ & $\begin{array}{l}\text { Apocrine } \\
(\mathrm{n}=4)\end{array}$ & $\begin{array}{l}\text { Metaplastic } \\
\quad(\mathrm{n}=1)\end{array}$ & $\begin{array}{l}L C A Z \\
(\mathrm{n}=6)\end{array}$ & $\begin{array}{l}\text { Prominent TILs } \\
(\mathrm{n}=6)\end{array}$ \\
\hline Median age (range), years & $43(28-78)$ & $37(28-78)$ & $64(52-75)$ & 51 & $61(39-67)$ & $35(29-58)$ \\
\hline $\begin{array}{l}\text { BRCA1 germline mutation } \\
\text { carrier }\end{array}$ & $9(23 \%)$ & $8(36 \%)$ & 0 & 0 & 0 & $1(17 \%)$ \\
\hline Median tumor size (range), cm & $2.9(1.2-8.5)$ & $3.0(1.2-8.5)$ & $1.7(1.4-2.5)$ & 2.5 & $3.1(2.1-3.7)$ & $2.8(2.5-3.5)$ \\
\hline \multicolumn{7}{|l|}{ Histologic grade ${ }^{\mathrm{a}}$} \\
\hline II & $4(10 \%)$ & $1(5 \%)$ & $2(50 \%)$ & 0 & $1(17 \%)$ & 0 \\
\hline III & $35(90 \%)$ & $21(95 \%)$ & $2(50 \%)$ & $1(100 \%)$ & $5(83 \%)$ & $6(100 \%)$ \\
\hline \multicolumn{7}{|l|}{ Nuclear grade $\mathrm{b}$} \\
\hline II & $3(8 \%)$ & $1(5 \%)$ & $2(50 \%)$ & 0 & 0 & 0 \\
\hline III & 36 (92\%) & 21 (95\%) & $2(50 \%)$ & $1(100 \%)$ & $6(100 \%)$ & $6(100 \%)$ \\
\hline Lymph node metastasis & $19(49 \%)$ & $11(50 \%)$ & $2(50 \%)$ & 0 & $2(33 \%)$ & $4(67 \%)$ \\
\hline $\begin{array}{l}\text { Median follow-up (range), } \\
\text { month }\end{array}$ & $62(10-143)$ & $58(24-143)$ & $81(53-117)$ & 71 & $47(10-122)$ & $66(32-137)$ \\
\hline Distant metastasis & $17(44 \%)$ & $9(41 \%)$ & $1(25 \%)$ & $1(100 \%)$ & $4(67 \%)$ & $2(33 \%)$ \\
\hline Lung & 11 & 7 & 0 & 0 & 3 & 1 \\
\hline Bone & 6 & 3 & 1 & 0 & 2 & 0 \\
\hline Brain & 5 & 3 & 0 & 1 & 0 & 1 \\
\hline Liver & 2 & 1 & 1 & 0 & 0 & 0 \\
\hline Other & 1 & 1 & 0 & 0 & 0 & 0 \\
\hline \multicolumn{7}{|l|}{ Survival } \\
\hline NED & $22(56 \%)$ & 13 (59\%) & 3 (75\%) & 0 & $2(33 \%)$ & 4 (67\%) \\
\hline AWD & $1(3 \%)$ & 0 & 0 & 0 & $1(17 \%)$ & 0 \\
\hline DOD & $16(41 \%)$ & $9(41 \%)$ & $1(25 \%)$ & $1(100 \%)$ & $3(50 \%)$ & $2(33 \%)$ \\
\hline
\end{tabular}

Abbreviations: AWD, alive with disease; DOD, died of disease; LCAZ, large central acellular zone; NED, no evidence of disease; NOS, not otherwise specified; TILs, tumor-infiltrating lymphocytes; TNBC, triple negative breast cancer.

${ }^{a}$ No histologic grade I tumor is present in this cohort.

${ }^{\mathrm{b}}$ No nuclear grade I tumor is present in this cohort.

\section{Triple Negative Breast Cancer Not Otherwise Specified}

Triple negative breast cancer not otherwise specified comprised the majority of the cases in our study $(22 / 39,56 \%)$. No statistically significant differences in age, tumor size, grade, lymph node involvement, distant metastases, or survival were seen between the triple negative breast cancer not otherwise specified group and the overall cases in our cohort. The average number of non-synonymous mutations per case in the triple negative breast cancer not otherwise specified group was 3.41 (range 0-10), significantly higher than that of the triple negative breast cancers in the Cancer Genome Atlas cohort (mean 2.37, range $0-7)(P=0.01567$, Fisher's exact test). The most frequently mutated genes in this group were TP53 (18 cases, 82\%) followed by MLL2 (3 cases, 14\%), mirroring the findings in our overall cohort. PIK3CA mutations, however, were infrequent in this group, limited to only one case (5\%). None of the above mutations was significantly different in frequency from that of the triple negative breast cancers in the Cancer Genome Atlas cohort. Mutations that reached statistical significance (despite having a lower mutation frequency than TP53 or MLL2) include JAK1, TBK1, and TGFBR2, each present in two $(9 \%)$ cases in our study and in none of the cases in the triple negative breast cancers in the Cancer Genome Atlas cohort $(P=0.047)$. The vast majority of the highest frequency copy number alterations (amplifications of MYC, MCL1, and DDR2) were seen in this group (Figure 4).

\section{Triple Negative Breast Cancer with Apocrine Differentiation}

Triple negative breast cancer with apocrine differentiation (apocrine triple negative breast cancer) comprised a small subset of the cases in our cohort $(4 / 39,10 \%)$. Patients with apocrine triple negative breast cancer were older (mean age 64 years) than patients with non-apocrine triple negative breast cancer (mean age 45 years) ( $P=0.01303$, two-tailed student $t$-test). Apocrine triple negative breast cancers had lower histologic and nuclear grade than non-apocrine triple negative breast cancers (Table 1). Specifically, $50 \%$ of the apocrine triple negative breast cancers had histologic grade II vs only $5 \%$ of the non-apocrine triple negative breast cancers $(P=0.04512$, Fisher's exact test); similarly $50 \%$ of apocrine triple negative breast cancers had nuclear grade II vs only $3 \%$ of the non-apocrine triple negative breast cancers $(P=0.02342$, Fisher's exact 

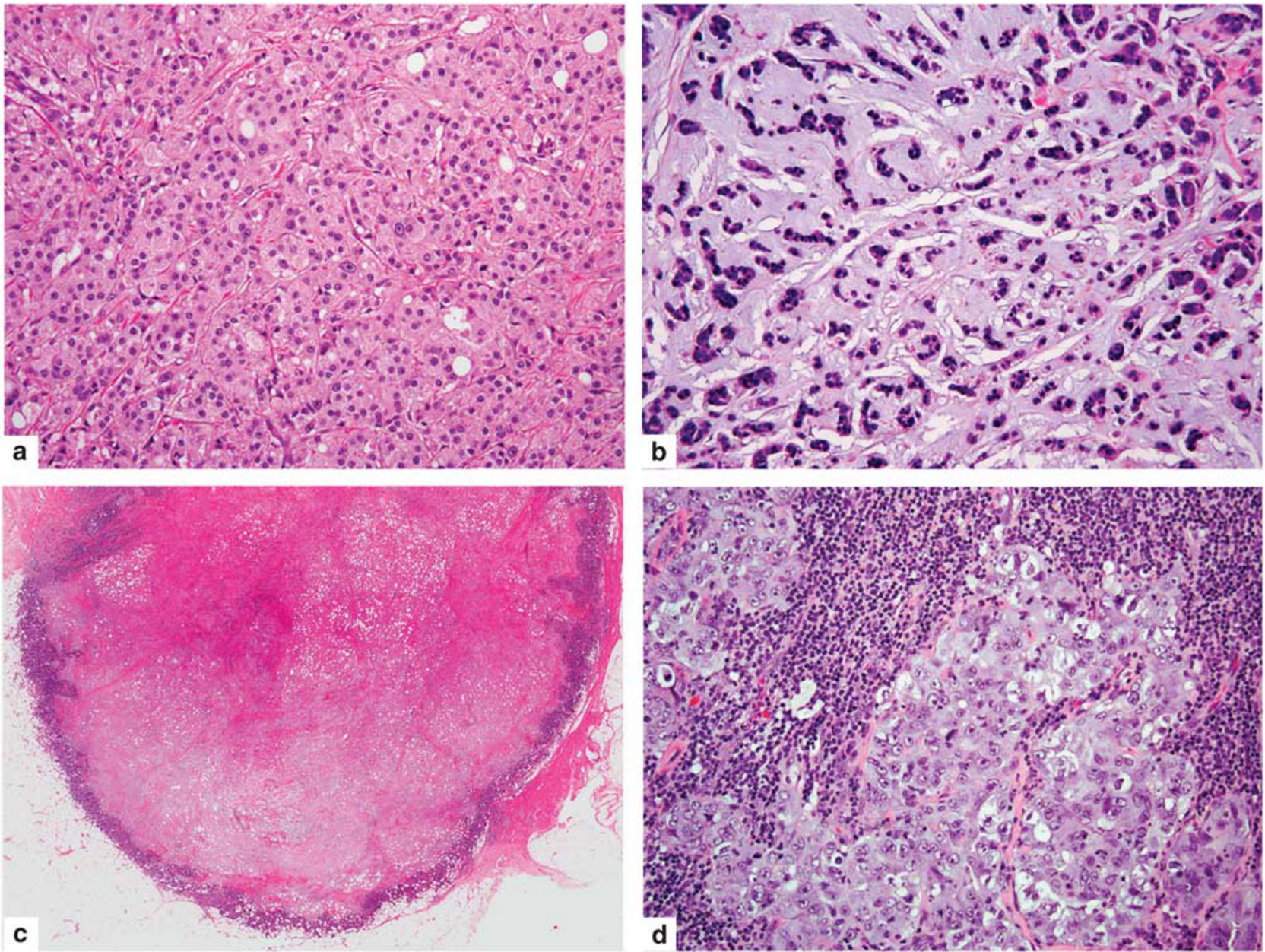

Figure 1 Special histologic subtypes and special morphologic features of triple negative breast cancers. (a) Carcinoma with apocrine differentiation (apocrine triple negative breast cancer); (b) metaplastic carcinoma; (c) tumor with a large central acellular zone of necrosis or fibrosis; (d) tumor with prominent tumor-infiltrating lymphocytes.

test). Patients with apocrine triple negative breast cancer also appeared to have smaller tumor size (median size $1.7 \mathrm{~cm}$, range $1.4-2.5 \mathrm{~cm}$ ), a lower rate of distant metastasis (one case, $25 \%$ ), and a lower rate of death from breast cancer (one case, 25\%); however, none of these differences reached statistical significance.

The average number of non-synonymous mutations per case in the apocrine triple negative breast cancer group was 4.5 (range 3-8), significantly higher than that of the triple negative breast cancers of the Cancer Genome Atlas cohort (mean 2.37, range 0-7, $P=0.03145$, Mann-Whitney U test). Apocrine triple negative breast cancers displayed a distinctive repertoire of somatic genetic alterations, characterized by less frequent TP53 mutations (one of four cases, $25 \%$ ) than in other morphologic subgroups of triple negative breast cancers from our cohort and from triple negative breast cancers from the Cancer Genome Atlas $(P=0.027$, Fisher's exact test $)$. The most commonly mutated genes in the apocrine triple negative breast cancer group were PIK3CA and NF1, each mutated in two cases (50\%). Although the relative frequencies of PIK3CA mutations in the apocrine triple negative breast cancer group and the triple negative breast cancers of the Cancer Genome Atlas cohort did not reach statistical significance, a trend was observed toward higher frequency of PIK3CA mutations in the apocrine triple negative breast cancer group (two cases (50\%) in apocrine triple negative breast cancer; seven cases $(9 \%)$ in triple negative breast cancers of the Cancer Genome Atlas cohort, $P=0.058$, Fisher's exact test). In addition, an apocrine triple negative breast cancer without PIK3CA mutation in our study showed a mutation in PIK3R1. Accordingly, if all activating mutations in PI3K pathway components are considered, apocrine triple negative breast cancers displayed significantly more frequent mutations affecting canonical genes of the PI3K pathway, including PIK3CD, PIK3R1, and AKT1, than triple negative breast cancers from the Cancer Genome Atlas study (three cases $(75 \%)$ of apocrine triple negative breast cancers vs eight cases $(10 \%)$ triple negative breast cancers of the Cancer Genome Atlas cohort, $P=0.0069$, Fisher's exact test). In the 

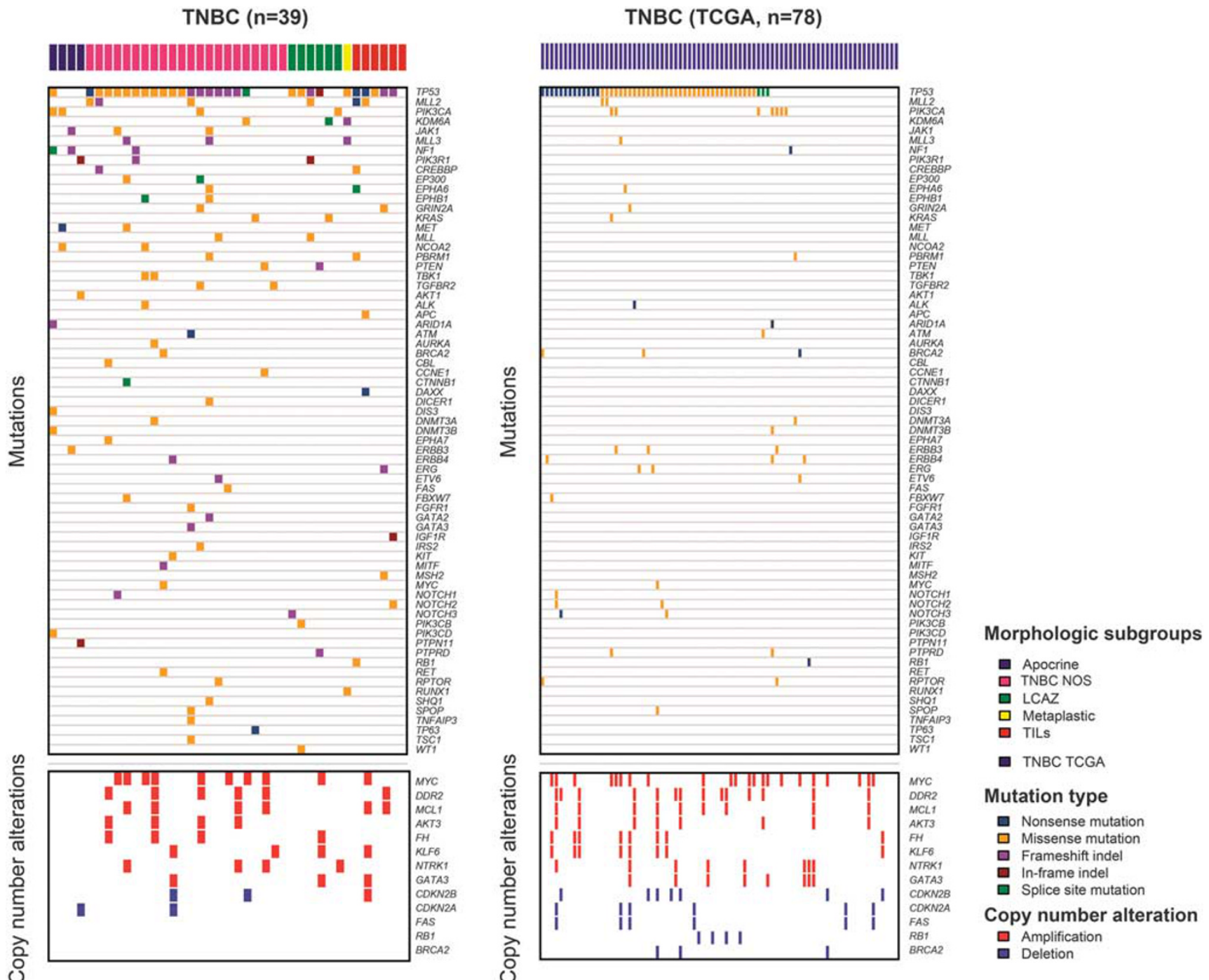

Figure 2 The landscape of somatic genetic alterations in triple negative breast cancers. Top panels: the repertoire of somatic nonsynonymous mutations in our triple negative breast cancer cohort as compared with the corresponding genes in triple negative breast cancers in the Cancer Genome Atlas Cohort. Bottom panels: the genes amplified in at least 10\% of our triple negative breast cancer cohort or deleted in at least $5 \%$ of our triple negative breast cancer cohort, as compared with the same genes in triple negative breast cancers in the Cancer Genome Atlas cohort. LCAZ, large central acellular zone; NOS, not otherwise specified; TCGA, the Cancer Genome Atlas; TNBC, triple negative breast cancer; TILs, prominent tumor-infiltrating lymphocytes.

immunohistochemical analysis of AR expression in apocrine triple negative breast cancers, all but one case expressed androgen receptor (Figure 5). The case that lacked androgen receptor expression was re-evaluated morphologically and the apocrine histologic features were confirmed (Figure 5c and d). This case harbored a PIK3CA mutation.

NF1 mutations were more frequently observed in the apocrine triple negative breast cancer group in our cohort than in triple negative breast cancers of the Cancer Genome Atlas cohort $(2 / 4,50 \%$ vs $2 / 78$, $3 \%, P=0.010$, Fisher's exact test). Other genes with significantly higher mutation frequency in the apocrine triple negative breast cancer group in our study included known cancer genes such as DIS3, JAK1, MET, NCOA2, and PTPN11, all of which were mutated in a single case each $(25 \%)$ in our apocrine triple negative breast cancer group and in none of the triple negative breast cancer cases in the Cancer Genome Atlas data set (all $P=0.047$, Fisher's exact tests).

Apocrine triple negative breast cancers had a lower level of genetic instability, with few amplifications and deletions (Figure 4). No recurrent copy number alterations were identified in this group.

\section{Metaplastic Carcinoma}

There was a single case of metaplastic carcinoma (matrix producing type) in this study. The tumor measured $2.5 \mathrm{~cm}$ and was high grade. Lymph nodes were not involved at presentation, but the patient developed brain metastasis and died of disease 

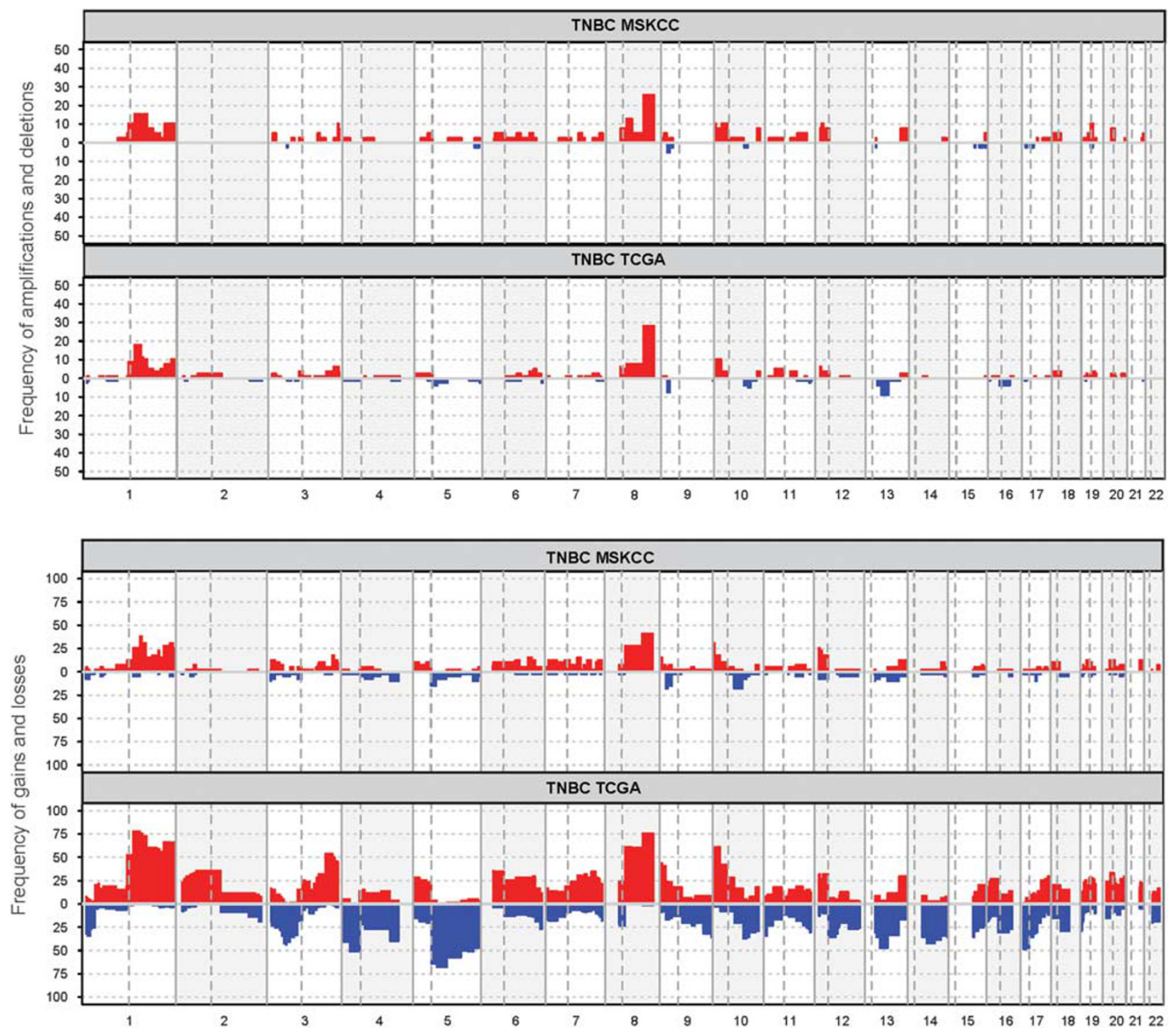

Figure 3 Frequency of copy number aberrations in our triple negative breast cancer cohort as compared with triple negative breast cancers in the Cancer Genome Atlas cohort. MSKCC, Memorial Sloan Kettering Cancer Center; TCGA, the Cancer Genome Atlas; TNBC, triple negative breast cancer.

71 months following breast cancer diagnosis. The number of non-synonymous mutations in this case was 3 , not different from that of the triple negative breast cancers from the Cancer Genome Atlas study (mean 2.37, range $0-7, P=0.4572$, Mann-Whitney $\mathrm{U}$ test). This tumor harbored mutations affecting TP53, KDM6A, and MLL3. A separate study of genetic alterations in a larger cohort of metaplastic carcinoma is ongoing.

\section{Triple Negative Breast Cancer with a Large Central Acellular Zone}

Six $(15 \%)$ triple negative breast cancers had a large central acellular zone. Patients with large central acellular zone tumors demonstrated a trend toward higher rates of distant metastasis (67\%) and death from disease $(50 \%)$, although it did not reach statistical significance. We found no statistically significant differences in age, tumor size, grade, or frequency of lymph node involvement between the triple negative breast cancer with a large central acellular zone group and other patients in our cohort. The average number of non-synonymous mutations per case in the large central acellular zone group was 2.5 (range 1-6), not significantly different from that of the triple negative breast cancers from the Cancer Genome Atlas study (mean 2.37. range 0-7, $P=0.2691$, Mann-Whitney U test). The most commonly mutated gene in the large central acellular zone group were TP53 (four cases, 67\%) followed by KDM6A (two cases, 33\%). We observed a trend toward a higher percentage of KDM6A 
Table 2 Comparison of genetic alterations by histologic subtypes and morphologic features

\begin{tabular}{|c|c|c|c|c|c|c|}
\hline & $\begin{array}{l}\text { All cases } \\
(\mathrm{n}=39)\end{array}$ & $\begin{array}{c}\text { TNBC NOS } \\
(\mathrm{n}=22)\end{array}$ & $\begin{array}{l}\text { Apocrine } \\
(\mathrm{n}=4)\end{array}$ & $\begin{array}{l}\text { Metaplastic } \\
\quad(\mathrm{n}=1)\end{array}$ & $\begin{array}{l}L C A Z \\
(\mathrm{n}=6)\end{array}$ & $\begin{array}{l}\text { Prominent TILs } \\
(\mathrm{n}=6)\end{array}$ \\
\hline $\begin{array}{l}\text { Average number of } \\
\text { mutations per case (range) }\end{array}$ & $3.33(0-10)$ & $3.41(0-10)$ & $4.5(3-8)$ & 3 & $2.5(1-6)$ & $3.17(0-6)$ \\
\hline Most frequent mutations & $\begin{array}{c}\text { TP53 }(74 \%) \\
\text { MLL2 (15\%) } \\
\text { PIK3CA }(10 \%)\end{array}$ & $\begin{array}{l}\text { TP53 }(82 \%) \\
\text { MLL2 }(14 \%)\end{array}$ & $\begin{array}{l}\text { PIK3CA }(50 \%) \\
\text { NF1 }(50 \%)\end{array}$ & $\begin{array}{c}\text { TP53 } \\
\text { KDM6A } \\
M L L 3\end{array}$ & $\begin{array}{c}\text { TP53 }(67 \%) \\
\text { KDM6A }(33 \%)\end{array}$ & $\begin{array}{l}\text { TP53 (83\%) } \\
\text { MLL2 (33\%) }\end{array}$ \\
\hline $\begin{array}{l}\text { Most frequent copy number } \\
\text { alterations (amplifications) }\end{array}$ & $\begin{array}{l}\text { MYC }(26 \%) \\
\text { MCL1 (15\%) } \\
\text { DDR2 (15\%) }\end{array}$ & $\begin{array}{l}\text { MYC }(36 \%) \\
\operatorname{MCL1}(23 \%)\end{array}$ & None & - & $\operatorname{ATM}(33 \%)$ & CCNE1 $(33 \%)$ \\
\hline
\end{tabular}

Abbreviations: LCAZ, large central acellular zone; NOS, not otherwise specified; TILs, tumor-infiltrating lymphocytes; TNBC, triple negative breast cancer.

mutations in the triple negative breast cancer with large central acellular zone group than in the triple negative breast cancer not otherwise specified group (triple negative breast cancer with a large central acellular zone: $2 / 6,33 \%$; triple negative breast cancer not otherwise specified: $2 / 22,9 \%$ ), although this difference did not reach statistical significance ( $P=0.191$, Fisher's exact test). In both groups, the frequency of KDM6A mutations was significantly higher than of that of triple negative breast cancers from the Cancer Genome Atlas study, which did not show any KDM6A mutations (large central acellular zone vs triple negative breast cancers in the Cancer Genome Atlas cohort, $P=0.004$; triple negative breast cancer not otherwise specified vs triple negative breast cancers in the Cancer Genome Atlas cohort, $P=0.047$, Fisher's exact tests). Two $(33 \%)$ of the cases in the large central acellular zone group had amplification of ATM - a significantly higher frequency than that of triple negative breast cancers in the Cancer Genome Atlas cohort (one case, 1\%; $P=0.012$, Fisher's exact test).

\section{Tumors with Prominent Tumor-infiltrating Lymphocytes}

Six $(15 \%)$ triple negative breast cancers had prominent tumor-infiltrating lymphocytes. Although patients in the prominent tumor-infiltrating lymphocytes group were younger than patients without prominent tumor-infiltrating lymphocytes (mean age 38.5 years vs 48.36 years; $P=0.1319$ ), this did not reach statistical significance. No statistically significant differences in tumor size, grade, lymph node involvement, distant metastases, or death from breast cancer were seen between the prominent tumorinfiltrating lymphocytes group and the other groups in this cohort. The average number of nonsynonymous mutations per case in the tumors with prominent tumor-infiltrating lymphocytes group was 3.17 (range 0-6), not significantly different from that of the triple negative breast cancers from the Cancer Genome Atlas study (mean 2.37, range 0-7, $P=0.3691$, Mann-Whitney U test). The genes most frequently mutated in triple negative breast cancer with prominent tumor-infiltrating lymphocytes group were TP53 (five cases, 83\%) and MLL2 (two cases, 33\%). None of the mutations in triple negative breast cancers with prominent tumor-infiltrating lymphocytes reached statistical significance as compared with the triple negative breast cancers in the Cancer Genome Atlas cohort. Recurrent amplifications in the prominent tumorinfiltrating lymphocytes group were seen in CCNE1 (two cases, 33\%) and MCL1 (two cases, 33\%). Of these, the amplification of CCNE1 was significantly more frequent in triple negative breast cancers with prominent tumor-infiltrating lymphocytes as compared with the triple negative breast cancers in the Cancer Genome Atlas cohort (two cases (33\%) and two cases $(2.6 \%)$, respectively; $P=0.024$, Fisher's exact test).

\section{Genetic Alterations in BRCA1 Germline Mutation Carriers}

Nine patients were BRCA1 germline mutation carriers. BRCA1 germline mutation carriers were younger than the other patients in our cohort (mean age 34.2 years vs 50.6 years; $P=0.002$ ). No statistically significant differences in tumor size, grade, lymph node involvement, distant metastases, or death from breast cancer were seen between the BRCA1 germline mutation carriers and the other patients in our study. Most $(8 / 9,89 \%)$ of BRCA1 germline mutation carriers had triple negative breast cancer not otherwise specified. One patient $(1 / 9,11 \%)$ had triple negative breast cancer with prominent tumor-infiltrating lymphocytes. The average number of non-synonymous mutations per case in BRCA1 germline mutation carriers' tumors was 4.44 (range 1-10) and was significantly different from that of the triple negative breast cancers from the Cancer Genome Atlas study (mean 2.37, range $0-7, P=0.0186$ ). The most frequently mutated gene in tumors from BRCA1 germline mutation carriers was TP53 (8 cases, 89\%), a frequency similar to that reported for triple negative breast cancers from the Cancer Genome Atlas cohort (64 cases, $82 \% ; P=1.00)$. 


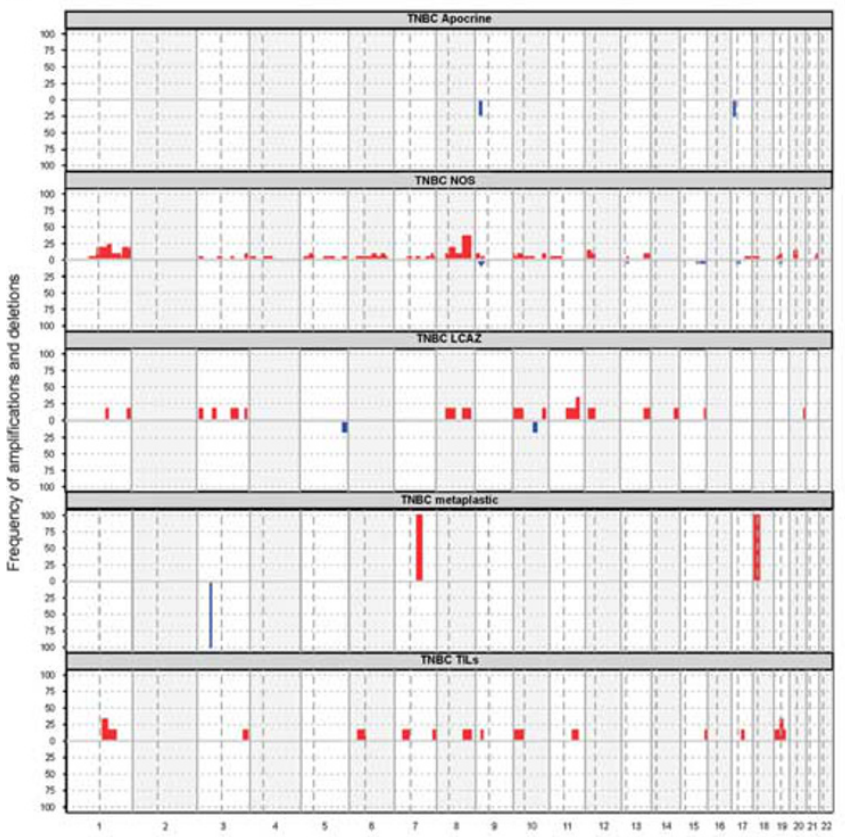

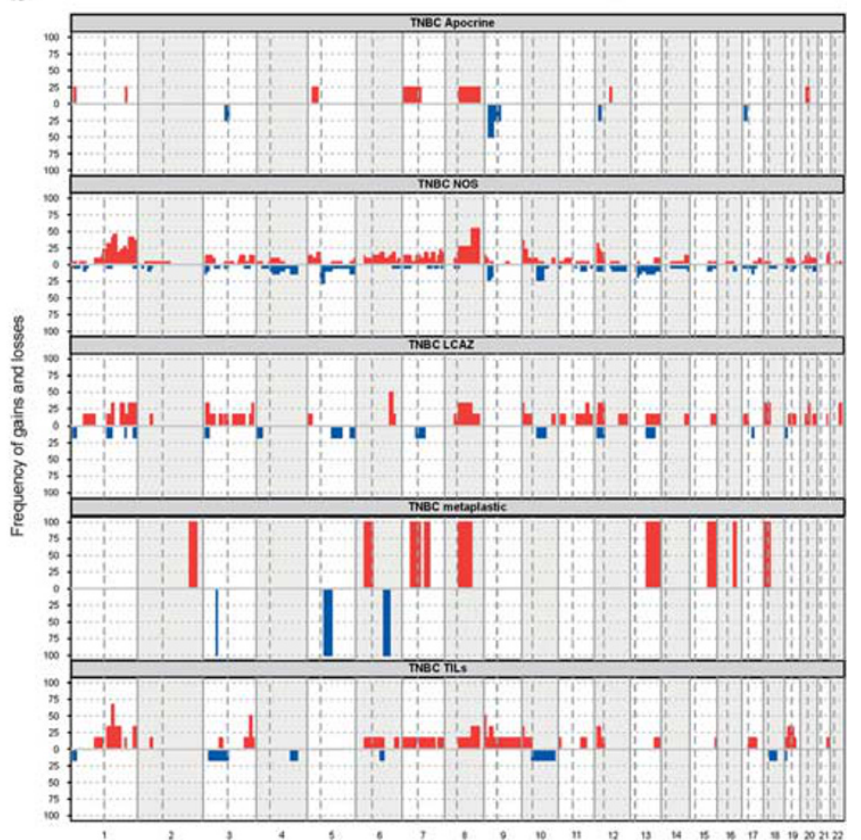

Figure 4 Frequency of copy number aberrations in our triple negative breast cancer cohort, by morphologic group. (a) Amplifications and deletions; (b) gains and losses. LCAZ, large central acellular zone; NOS, not otherwise specified; TNBC, triple negative breast cancer; TILs, prominent tumor-infiltrating lymphocytes.

\section{Discussion}

Although the genomic landscape of triple negative breast cancers is heterogeneous and complex, here, we show that many of the key somatic genetic alterations in triple negative breast cancers elucidated by studies using large scale genomic analysis techniques can be detected using a targeted next-generation sequencing assay (MSK-IMPACT) in routine clinical use at our institution. ${ }^{16}$ Moreover, we show that the histologic analysis of triple negative breast cancers can provide useful information regarding the predicted somatic genetic landscape of individual triple negative breast cancer tumors. For instance, mutations in TP53 and PIK3CA are known to predominate in triple negative breast cancers, being reported in approximately $80 \%$ and $10 \%$ of triple negative breast cancers, respectively. ${ }^{13,14}$ We found similar mutation frequencies in our cohort, in which TP53 and PIK3CA mutations were present in $74 \%$ and $10 \%$ of cases, respectively. However, these findings did not apply to all morphologic subtypes of triple negative breast cancer. For example, activating mutations in both PIK3CA as well as other key PI3K pathway components were enriched within the apocrine triple negative breast cancer group $(75 \%$ of cases), a subset of triple negative breast cancers found to express androgen receptor more frequently than other forms of triple negative breast cancer. Lehmann et $a l^{40}$ also found PIK3CA mutations in $40 \%$ of their luminal androgen receptor tumors, a molecular subtype thought to be enriched in triple negative breast cancer with apocrine morphology, but did not correlate the findings with histologic analysis. Although our study did not include transcriptomic profiling analysis, we speculate that triple negative breast cancers that express androgen receptor in our study would strongly correlate with the luminal androgen receptor subtype described by Lehmann et $a l^{41}$ and Burstein et al, ${ }^{42}$ because it has been reported that androgen receptor mRNA was highly expressed in the luminal androgen receptor subtype, much relative to all other subtypes. ${ }^{41}$ To the best of our knowledge, our study is the first comprehensive analysis of apocrine triple negative breast cancers as defined by histologic features. Our study also demonstrated that, relative to the triple negative breast cancers from the Cancer Genome Atlas dataset, apocrine triple negative breast cancers displayed a significantly higher rate of PI3K pathway mutations and NF1 mutations and a significantly lower rate of TP53 mutations and copy number aberrations. The high frequency of PI3K pathway mutations in apocrine triple negative breast cancer is more similar to that in luminal types of breast cancers.

For the mutations targeted by our assay, we observed a significantly higher number of somatic non-synonymous mutations per case in our triple negative breast cancer cohort as compared with that reported in the triple negative breast cancers in the Cancer Genome Atlas dataset. This is likely due to the higher depth of coverage and more sensitive detection that is possible with the MSK-IMPACT platform as compared to the whole exome sequence 

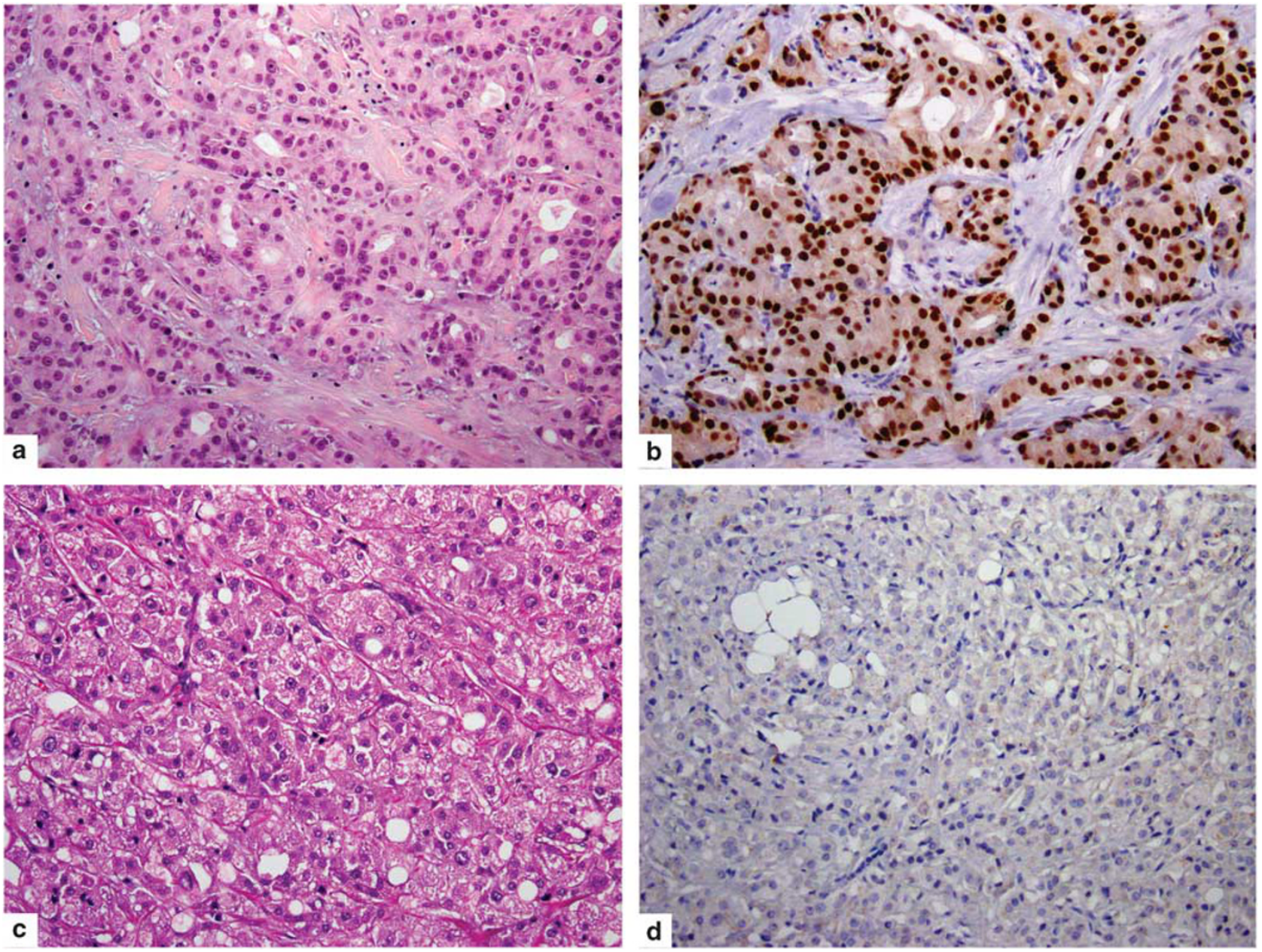

Figure 5 Immunohistochemical staining for androgen receptor in triple negative breast cancer with apocrine differentiation (apocrine triple negative breast cancer). (a and b) An example of androgen receptor-positive apocrine triple negative breast cancer; H\&E and androgen receptor immunohistochemical stain; (c and d) The apocrine triple negative breast cancer that was androgen receptor-negative but harbored a PIK3CA mutation; H\&E and androgen receptor immunohistochemical stain.

analysis used by the TCGA study, given that we achieved a coverage of at least $77 \%$ (tumors: median 98\%, range 84-99\%; normal: median 96\%, range $77-98 \%)$ at $100 \times$ depth compared with the Cancer Genome Atlas requirement of including samples with at least $70 \%$ coverage at $20 \times$ depth. Additionally, we used MuTect for calling SNVs, which has been shown to be more sensitive than VarScan 2 and SomaticSniper used by the Cancer Genome Atlas. ${ }^{43-45}$ As the genes targeted by our assay include actionable and potentially actionable targets, these results are of particular interest with regard to the treatment of triple negative breast cancers as a lack of recurrent actionable targets is the basis for the reliance on cytotoxic agents as a means for systemic therapy.

This study has several limitations. First, the relatively small number of cases in each morphologic group renders the analysis performed exploratory and hypothesis-generating. Second, we have surveyed the presence of somatic genetic alterations affecting 229 genes. Hence, we cannot exclude the possibility that additional differences between specific subtypes of triple negative breast cancer may be present if whole exome or whole genome sequencing was performed. In fact, rare subtypes of triple negative breast cancer, such as adenoid cystic carcinomas, have been shown to be driven by recurrent MYB-NFIB fusion genes. ${ }^{9}$ Nevertheless, as a proof-of-concept study, our findings demonstrate that a next-generation sequencing panel (MSK-IMPACT) can reproduce findings similar to those obtained by genomic studies carried out on a much larger scale ${ }^{13,14}$ and that a careful morphologic analysis of triple negative breast cancers can provide useful information regarding the somatic genetic composition of individual tumors. Furthermore, our findings suggest that triple negative breast cancers with apocrine differentiation likely display a landscape of somatic genetic alterations distinct from that of other triple negative breast cancers. Given these observations, further studies dissecting the genomic landscape of specific subsets of triple negative breast cancers are warranted. 


\section{Acknowledgments}

This study was supported by Farmer Family Foundation. SP is funded by a Susan G Komen Postdoctoral Fellowship Grant (PDF14298348). Research reported in this publication was supported in part by a Cancer Center Support Grant of the National Institutes of Health/National Cancer Institute (Grant No. P30CA008748). The content is solely the responsibility of the authors and does not necessarily represent the official views of the National Institutes of Health. We thank Dr Kiran Jakate for her assistance.

\section{Disclosure/conflict of interest}

The authors declare no conflict of interest.

\section{References}

1 Dent R, Trudeau M, Pritchard KI et al. Triple-negative breast cancer: clinical features and patterns of recurrence. Clin Cancer Res 2007;13:4429-4434.

2 Livasy CA, Karaca G, Nanda R et al. Phenotypic evaluation of the basal-like subtype of invasive breast carcinoma. Mod Pathol 2006;19:264-271.

3 Thike AA, Cheok PY, Jara-Lazaro AR et al. Triplenegative breast cancer: clinicopathological characteristics and relationship with basal-like breast cancer. Mod Pathol 2010;23:123-133.

4 Weigelt B, Kreike B, Reis-Filho JS. Metaplastic breast carcinomas are basal-like breast cancers: a genomic profiling analysis. Breast Cancer Res Treat 2009;117: 273-280.

5 Geyer FC, Weigelt B, Natrajan R et al. Molecular analysis reveals a genetic basis for the phenotypic diversity of metaplastic breast carcinomas. J Pathol 2010;220:562-573.

6 Vranic S, Tawfik O, Palazzo J et al. EGFR and HER-2/ neu expression in invasive apocrine carcinoma of the breast. Mod Pathol 2010;23:644-653.

7 Farmer P, Bonnefoi $\mathrm{H}$, Becette $\mathrm{V}$ et al. Identification of molecular apocrine breast tumours by microarray analysis. Oncogene 2005;24:4660-4671.

8 Wetterskog D, Lopez-Garcia MA, Lambros MB et al. Adenoid cystic carcinomas constitute a genomically distinct subgroup of triple-negative and basal-like breast cancers. J Pathol 2012;226:84-96.

9 Martelotto LG, De Filippo MR, Ng CK et al. Genomic landscape of adenoid cystic carcinoma of the breast. J Pathol 2015;237:179-189.

10 Diallo R, Schaefer KL, Bankfalvi A et al. Secretory carcinoma of the breast: a distinct variant of invasive ductal carcinoma assessed by comparative genomic hybridization and immunohistochemistry. Hum Pathol 2003;34:1299-1305.

11 Piscuoglio S, Hodi Z, Katabi N et al. Are acinic cell carcinomas of the breast and salivary glands distinct diseases? Histopathology 2015;67:529-537.

12 Guerini-Rocco E, Hodi Z, Piscuoglio S et al. The repertoire of somatic genetic alterations of acinic cell carcinomas of the breast: an exploratory, hypothesisgenerating study. J Pathol 2015;237:166-178.
13 Cancer Genome Atlas N. Comprehensive molecular portraits of human breast tumours. Nature 2012;490: 61-70.

14 Shah SP, Roth A, Goya $\mathrm{R}$ et al. The clonal and mutational evolution spectrum of primary triplenegative breast cancers. Nature 2012;486:395-399.

$15 \mathrm{Ng}$ CK, Schultheis AM, Bidard FC et al. Breast cancer genomics from microarrays to massively parallel sequencing: paradigms and new insights. J Natl Cancer Inst 2015;107:pii: djv015.

16 Cheng DT, Mitchell TN, Zehir A et al. Memorial Sloan Kettering-Integrated Mutation Profiling of Actionable Cancer Targets (MSK-IMPACT): a hybridization capture-based next-generation sequencing clinical assay for solid tumor molecular oncology. J Mol Diagn 2015;17:251-264.

17 Won HH, Scott SN, Brannon AR et al. Detecting somatic genetic alterations in tumor specimens by exon capture and massively parallel sequencing. J Vis Exp 2013;80:e50710.

18 Hammond ME, Hayes DF, Dowsett M et al. American Society of Clinical Oncology/College of American Pathologists guideline recommendations for immunohistochemical testing of estrogen and progesterone receptors in breast cancer. Arch Pathol Lab Med 2010;134:907-922.

19 Hammond ME, Hayes DF, Dowsett M et al. American Society of Clinical Oncology/College Of American Pathologists guideline recommendations for immunohistochemical testing of estrogen and progesterone receptors in breast cancer. J Clin Oncol 2010;28: 2784-2795.

20 Wolff AC, Hammond ME, Hicks DG et al. Recommendations for human epidermal growth factor receptor 2 testing in breast cancer: American Society of Clinical Oncology/College of American Pathologists clinical practice guideline update. Arch Pathol Lab Med 2014;138:241-256.

21 Wolff AC, Hammond ME, Hicks DG et al. Recommendations for human epidermal growth factor receptor 2 testing in breast cancer: American Society of Clinical Oncology/College of American Pathologists clinical practice guideline update. J Clin Oncol 2013;31: 3997-4013.

22 Reis-Filho JS, Lakhani SR, Gobbi H et al. Metaplastic carcinoma. In: Lakhani SR, Ellis IO, Schnitt SJ et al. (eds). WHO Classification of Tumours of the Breast. International Agency for Research on Cancer (IARC): Lyon, France. 2012, pp 8-52.

23 O'Malley F, Eusebi V, Lakhani SR. Carcinomas with apocrine differentiation. In: Lakhani S, Ellis IO, Schnitt SJ et al. (eds). WHO Classification of Tumours of the Breast. International Agency for Research on Cancer (IARC): Lyon, France. 2012, pp 3-54.

24 Tsuda H, Takarabe T, Hasegawa $\mathrm{F}$ et al. Large, central acellular zones indicating myoepithelial tumor differentiation in high-grade invasive ductal carcinomas as markers of predisposition to lung and brain metastases. Am J Surg Pathol 2000;24:197-202.

25 Salgado R, Denkert C, Demaria S et al. The evaluation of tumor-infiltrating lymphocytes (TILs) in breast cancer: recommendations by an International TILs Working Group 2014. Ann Oncol 2015;26:259-271.

26 Loi S, Sirtaine N, Piette F et al. Prognostic and predictive value of tumor-infiltrating lymphocytes in a phase III randomized adjuvant breast cancer trial in node-positive breast cancer comparing the addition of 
docetaxel to doxorubicin with doxorubicin-based chemotherapy: BIG 02-98. J Clin Oncol 2013;31:860-867.

27 Gnirke A, Melnikov A, Maguire J et al. Solution hybrid selection with ultra-long oligonucleotides for massively parallel targeted sequencing. Nat Biotechnol 2009;27: 182-189.

28 Li H, Durbin R. Fast and accurate short read alignment with Burrows-Wheeler transform. Bioinformatics 2009;25:1754-1760.

29 DePristo MA, Banks E, Poplin R et al. A framework for variation discovery and genotyping using next-generation DNA sequencing data. Nat Genet 2011;43:491-498.

30 Cibulskis K, Lawrence MS, Carter SL et al. Sensitive detection of somatic point mutations in impure and heterogeneous cancer samples. Nat Biotechnol 2013;31:213-219.

31 Robinson JT, Thorvaldsdottir H, Winckler W et al. Integrative genomics viewer. Nat Biotechnol 2011;29: 24-26.

32 Carter H, Chen S, Isik L et al. Cancer-specific highthroughput annotation of somatic mutations: computational prediction of driver missense mutations. Cancer Res 2009;69:6660-6667.

33 Shihab HA, Gough J, Cooper DN et al. Predicting the functional, molecular, and phenotypic consequences of amino acid substitutions using hidden Markov models. Hum Mutat 2013;34:57-65.

34 Reva B, Antipin Y, Sander C. Predicting the functional impact of protein mutations: application to cancer genomics. Nucleic Acids Res 2011;39:e118.

35 Schwarz JM, Rodelsperger C, Schuelke M et al. MutationTaster evaluates disease-causing potential of sequence alterations. Nat Methods 2010;7:575-576.

36 Adzhubei IA, Schmidt S, Peshkin L et al. A method and server for predicting damaging missense mutations. Nat Methods 2010;7:248-249.
37 Choi Y, Sims GE, Murphy S et al. Predicting the functional effect of amino acid substitutions and indels. PLoS One 2012;7:e46688.

38 Weinreb I, Piscuoglio S, Martelotto LG et al. Hotspot activating PRKD1 somatic mutations in polymorphous low-grade adenocarcinomas of the salivary glands. Nat Genet 2014;46:1166-1169.

39 Ellis I, Collins L, Ichihara S et al. Invasive carcinoma of no special type. In: Lakhani S, Ellis IO, Schnitt SJ et al. WHO Classification of Tumours of the Breast. International Agency for Research on Cancer (IARC): Lyon, France. 2012, pp 34-38.

40 Lehmann BD, Bauer JA, Schafer JM et al. PIK3CA mutations in androgen receptor-positive triple negative breast cancer confer sensitivity to the combination of PI3K and androgen receptor inhibitors. Breast Cancer Res 2014;16:406.

41 Lehmann BD, Bauer JA, Chen X et al. Identification of human triple-negative breast cancer subtypes and preclinical models for selection of targeted therapies. J Clin Invest 2011;121:2750-2767.

42 Burstein MD, Tsimelzon A, Poage GM et al. Comprehensive genomic analysis identifies novel subtypes and targets of triple-negative breast cancer. Clin Cancer Res 2015;21:1688-1698.

43 Wang Q, Jia P, Li F et al. Detecting somatic point mutations in cancer genome sequencing data: a comparison of mutation callers. Genome Med 2013;5:91.

44 Alioto TS, Buchhalter I, Derdak S et al. A comprehensive assessment of somatic mutation detection in cancer using whole-genome sequencing. Nat Commun 2015;6:10001.

$45 \mathrm{Xu} \mathrm{H}$, DiCarlo J, Satya RV et al. Comparison of somatic mutation calling methods in amplicon and whole exome sequence data. BMC Genomics 2014;15:244.

Supplementary Information accompanies the paper on Modern Pathology website (http://www.nature.com/ modpathol) 